\title{
A probable giant planet imaged in the $\beta$ Pictoris disk ${ }^{\star}$ VLT/NaCo deep $L^{\prime}$-band imaging
}

\author{
A.-M. Lagrange ${ }^{1}$, D. Gratadour ${ }^{2}$, G. Chauvin ${ }^{1}$, T. Fusco $^{3}$, D. Ehrenreich ${ }^{1}$, D. Mouillet ${ }^{1}$, G. Rousset ${ }^{2,3}$, D. Rouan ${ }^{2}$, \\ F. Allard ${ }^{4}$, É. Gendron ${ }^{2}$, J. Charton ${ }^{1}$, L. Mugnier ${ }^{3}$, P. Rabou ${ }^{1}$, J. Montri ${ }^{3}$, and F. Lacombe ${ }^{2}$ \\ 1 Laboratoire d'Astrophysique de l'Observatoire de Grenoble, Université Joseph Fourier, CNRS (UMR 5571), BP 53, \\ 38041 Grenoble, France \\ e-mail: anne-marie.lagrange@obs.ujf-grenoble.fr \\ 2 Laboratoire d'Études Spatiales et d'Instrumentation en Astrophysique, Observatoire de Paris, CNRS (UMR 8109), \\ Université Pierre et Marie Curie, Université Paris-Diderot, 5 place Jules Janssen, 92190 Meudon, France \\ 3 Office National d'Études et de Recherches Aérospatiales, 29 avenue de la Division Leclerc, 92322 Châtillon, France \\ ${ }^{4}$ Centre de Recherche Astronomique de Lyon, CNRS (UMR 5574), Université Claude Bernard, École Normale Supérieure de Lyon, \\ 46 allée d'Italie, 69364 Lyon Cedex 7, France
}

Received 10 November 2008 / Accepted 18 November 2008

\section{ABSTRACT}

\begin{abstract}
Context. Since the discovery of its dusty disk in 1984, $\beta$ Pictoris has become the prototype of young early-type planetary systems, and there are now various indications that a massive Jovian planet is orbiting the star at $\sim 10 \mathrm{AU}$. However, no planets have been detected around this star so far.

Aims. Our goal was to investigate the close environment of $\beta$ Pic, searching for planetary companion(s).

Methods. Deep adaptive-optics $L^{\prime}$-band images of $\beta$ Pic were recorded using the NaCo instrument at the Very Large Telescope.

Results. A faint point-like signal is detected at a projected distance of $\simeq 8 \mathrm{AU}$ from the star, within the northeastern extension of the dust disk. Various tests were made to rule out possible instrumental or atmospheric artefacts at a good confidence level. The probability of a foreground or background contaminant is extremely low, based in addition on the analysis of previous deep HST images. Its $L^{\prime}=11.2$ apparent magnitude would indicate a typical temperature of $\sim 1500 \mathrm{~K}$ and a mass of $\sim 8 M_{\text {Jup }}$. If confirmed, it could explain the main morphological and dynamical peculiarities of the $\beta$ Pic system. The present detection is unique among A-stars by the proximity of the resolved planet to its parent star. Its closeness and location inside the $\beta$ Pic disk suggest a formation process by core accretion or disk instabilities rather than binary-like formation processes.
\end{abstract}

Key words. instrumentation: adaptive optics - stars: early-type - stars: planetary systems - stars: individual: $\beta$ Pic

\section{Introduction}

Understanding planetary systems' formation and evolution has become one of the biggest challenges in astronomy, since the imaging of a debris disk around $\beta$ Pictoris in the 80's (Smith \& Terrile 1984) and the discovery of the first exoplanet around the solar-like star 51 Pegasi during the 90's (Mayor \& Queloz 1995). While about 20 debris disks - disks containing dust that is not primordial but produced by collisions among larger rocky bodies - have been resolved at optical wavelengths today, $\beta$ Pic, an A5V star at a distance of $19.3 \pm 0.2$ pc (Crifo et al. 1997), remains the best studied young (12 -4 Myr; Zuckerman et al. 2001) system, with an impressive amount of indirect signs pointing toward the presence of planets.

The disk's inner part is relatively free of matter inside 50 astronomical units (AU). Lecavelier des Etangs et al. (1995) presented intriguing light variations possibly due to disk inhomogeneities produced by a Jupiter-size planet at $>6$ AU. Several asymmetries have been identified in the disk at optical

\footnotetext{
* Based on observations collected at the European Southern Observatory, Chile, ESO (runs 072.C-0624(B) and 60.A-9026(A)) and on observations made with the Space Telescope Imaging Spectrograph onboard the NASA/ESA Hubble Space Telescope.
}

(Kalas \& Jewitt 1995; Heap et al. 2000) and infrared (Telesco et al. 2005) wavelengths, as well as a warp at $~ 50 \mathrm{AU}$ (Mouillet et al. 1997; Heap et al. 2000). The structure is reproduced well by the deformation induced on colliding planetesimals by a giant planet on a slightly inclined orbit within $50 \mathrm{AU}$ of the star (Krist et al. 1996; Mouillet et al. 1997; Gorkavyi et al. 2004; Augereau et al. 2001; Thébault \& Beust 2001). Silicate dust is observed as circumstellar rings at 6,16 , and $30 \mathrm{AU}$ from the star (Okamoto et al. 2004), which could be explained by the presence of a 2-5-Jovian-mass $\left(M_{\mathrm{Jup}}\right)$ planet at $\sim 10$ AU from the star (Freistetter et al. 2007). Evaporating star-grazing comets have been identified (see, e.g., Lagrange et al. 2000, for a review) and dynamical simulations have shown that the gravitational perturbation of at least one giant planet at $\sim 10 \mathrm{AU}$ can account for the observed rate of evaporating bodies (Beust \& Morbidelli 2000); however, no planets have been detected so far, either through direct imaging or through radial velocity studies, due to the instrumental limitations of both techniques (Galland et al. 2006). In particular, the high spatial resolution imaging detection capabilities have so far been limited to distances typically of $\gtrsim 15-20$ AU.

We used the NAOS-CONICA instrument ( $\mathrm{NaCo})$, installed on the Very Large Telescope UT4 (Yepun) set in Paranal (Chile), to benefit from both the high image quality provided by the 
Table 1. Observing Log and companion position and flux relative to $\beta$ Pic for the 3 data set (A-C).

\begin{tabular}{|c|c|c|c|c|c|c|c|c|c|c|c|c|}
\hline \multirow[b]{2}{*}{ Set } & \multirow[b]{2}{*}{ Star } & \multirow[b]{2}{*}{ Date } & \multirow[b]{2}{*}{$\begin{array}{l}\text { DIT } \\
(\mathrm{s})\end{array}$} & \multirow[b]{2}{*}{ NDIT } & \multirow[b]{2}{*}{$\begin{array}{l}t_{\exp } \\
(\mathrm{s})\end{array}$} & \multirow[b]{2}{*}{$\begin{array}{l}\pi^{3} \\
(\mathrm{o})\end{array}$} & \multirow[b]{2}{*}{$\sec z$} & \multirow[b]{2}{*}{$\begin{array}{l}\langle E C\rangle^{4} \\
(\%)\end{array}$} & \multirow[b]{2}{*}{$\begin{array}{l}\left\langle\tau_{0}\right\rangle^{5} \\
(\mathrm{~ms})\end{array}$} & \multicolumn{3}{|c|}{ COMPANION } \\
\hline & & & & & & & & & & $\begin{array}{l}\text { Separation } \\
\text { (mas) }\end{array}$ & $\begin{array}{l}\text { PA } \\
\text { (o) }\end{array}$ & $\begin{array}{l}\Delta L^{\prime} \\
(\mathrm{mag})\end{array}$ \\
\hline \multirow[t]{2}{*}{$\mathrm{A}$} & $\beta$ Pic & $2003-11-10$ & 0.175 & 200 & 665 & $-18 /-9$ & $1.125 / 1.119$ & 62.71 & 11.47 & $411 \pm 8$ & $31.8 \pm 1.3$ & $7.7 \pm 0.3$ \\
\hline & HR 2435 & $2003-11-10$ & 0.175 & 200 & 630 & $-20 /-12$ & $1.148 / 1.140$ & 63.06 & 10.65 & & & \\
\hline \multirow[t]{2}{*}{$\bar{B}$} & $\beta$ Pic & $2003-11-10$ & 0.175 & 100 & 420 & $-27 /-19$ & $1.138 / 1.127$ & 57.94 & 8.08 & $411 \pm 8$ & $31.5 \pm 1.3$ & $7.9 \pm 0.4$ \\
\hline & HR 2435 & 2003-11-10 & 0.175 & 100 & 420 & $-28 /-20$ & $1.161 / 1.149$ & 60.23 & 9.70 & & & \\
\hline \multirow[t]{2}{*}{$\overline{\mathrm{C}}$} & $\beta$ Pic & $2003-11-13$ & 0.175 & 200 & 385 & $-24 /-19$ & $1.161 / 1.149$ & 46.94 & 4.50 & $401 \pm 8$ & $32.1 \pm 1.4$ & $7.6 \pm 0.4$ \\
\hline & HR 2435 & $2003-11-13$ & 0.175 & 200 & 350 & $-22 /-17$ & $1.150 / 1.144$ & 44.99 & 2.72 & & & \\
\hline
\end{tabular}

${ }^{3}$ Range of parallactic angles at the start/end of the observation; ${ }^{4}$ the average coherent energy as estimated online by AO; ${ }^{5}$ the average coherence time as estimated online by $\mathrm{AO}$.

Nasmyth Adaptive Optics System (NAOS; Rousset et al. 2003) at infrared wavelengths and the good dynamics offered by the Near-Infrared Imager and Spectrograph (CONICA; Lenzen et al. 2003) detector, in order to study the immediate circumstellar environment of $\beta$ Pic.

\section{Observations and data reduction procedures}

\subsection{Observations}

The $L^{\prime}$-band images of $\beta$ Pic $\left(V=3.8, L^{\prime}=3.5\right)$ were obtained between 2003 November 10 and 2003 November 17 with $\mathrm{NaCo}$. The visible wavefront sensor was used with the $14 \times 14$ lenslet array, together with the visible dichroic. We used the CONICA L27 camera, which provides a pixel scale of $\sim 27$ mas. Saturated images of $\beta$ Pic were recorded, with detector integration times (DITs) of $0.175 \mathrm{~s}$ and a number of integrations (NDIT) of 100 or 200. Every two exposures ${ }^{1}$, spatial offsets were applied in order to allow sky and instrumental background removal. Unsaturated images were also recorded to get images of the stellar point spread function (PSF), as well as a photometric calibration. In this case, we added the long neutral density filter (transmission 0.018) in the CONICA optical path, and recorded images with DITs of $0.4 \mathrm{~s}$.

In addition, the binary IDS 22141S3712 (separation $\rho=$ $6630 \pm 10$ mas, position angle PA $=302.06 \pm 0.07^{\circ}$; van Dessel \& Sinachopoulos 1993) was observed on November 11 as an astrometric calibrator. A mean plate scale of $27.105 \pm 0.041$ mas and a true north orientation of $-0.10 \pm 0.07^{\circ}$ were derived and used to calibrate all $\beta$ Pic images. Saturated and unsaturated exposures were taken on the reference star HR 2435 (A0II, $V=4.4$ ). The purpose is to correct for the star halo (the wings of the PSF) present in the saturated exposures. To optimize the removal of any fixed speckle, $\beta$ Pic and HR 2435 were observed at close parallactic angles as much as possible. Finally, twilight flat fields were also recorded in $L^{\prime}$ band.

The observing conditions varied from exceptional (coherent energy ${ }^{2} E C>70 \%$, coherent time $\tau_{0}>20 \mathrm{~ms}$ ) to reasonable $\left(E C \sim 50 \%, \tau_{0}\right.$ of a few $\left.\mathrm{ms}\right)$, and sometimes poor ( $E C \sim 20-35 \%, \tau_{0} \sim 1-2 \mathrm{~ms}$ ), over the run, and the data quality varies accordingly. The best data set on $\beta$ Pic was obtained on November 10 . In the following, we describe the data reduction and analysis of three sets of data: (A) the very best set obtained on November 10; (B) a set of data with slightly poorer image

\footnotetext{
1 An exposure is completed after DIT $\times$ NDIT.

2 Since it is not possible to measure the Strehl ratio on our saturated data, we can only use the information provided by NAOS to assess the image quality. The coherent energies are those measured by the system in $K$ band.
}

quality obtained on the same night, with a shorter total exposure time, and (C) a set of data with poorer image quality obtained on November 13. However, Set $C$ is representative of the best data obtained in the nights following November 10. The instrumental configurations and the status of image quality for these three data sets can be found in Table 1 .

\subsection{Data processing}

The first step in data analysis was the cosmetic correction (badpixels, flat-fielding, background subtraction) and recentering of individual offset positions of $\beta$ Pic and HR 2435 observations. A first method was to directly apply standard routines from the eclipse library (Devillard 1997), using classical crosscorrelation algorithm. Special care was taken to estimate the background at each given position by averaging images obtained at the previous and successive offset positions in the observing sequence. Alternatively, a second method was used with improved software dedicated to adaptive optics (AO) image processing (see Gratadour et al. 2005). Following a different approach for the background subtraction, images at individual offset positions were recentered using a maximum likelihood algorithm at the level of a tenth of a pixel or better. The same overall process was used for both the object and the reference. Consistent results were found in terms of recentering precision and background subtraction (at less than the backgroung noise of $0.9 \mathrm{ADU})$.

As a second step, three parallel approaches were followed to study the close environment of $\beta$ Pic:

- A first approach consists in removing the PSF wings from the saturated images of $\beta$ Pic by a simple minimization of the residuals. To do so, we first divided $\beta$ Pic images by the ones for HR 2435 obtained under similar conditions (same DIT, NDIT, and pupil position). We then computed the scaling factor to be applied to the reference in order to scale its flux to that of $\beta$ Pic. We then subtracted the reference images to the $\beta$ Pic ones. Recentering and scaling processes were repeated to minimize the residuals with respective precisions of subpixels and 5\%. Tests were performed using rotated images of $\beta$ Pic for PSF subtraction. However, that PSF halo is not centro-symmetric and that the static aberrations are not overlaid worsen the subtraction process.

- The second approach is to follow the same subtraction sequence but to apply the maximum likelihood algorithm of Gratadour et al. (2005, see Fig. 1). The algorithm is used here to recenter the reference star with $\beta$ Pic and therefore confirm the previous estimation. Similar precision is achieved. 

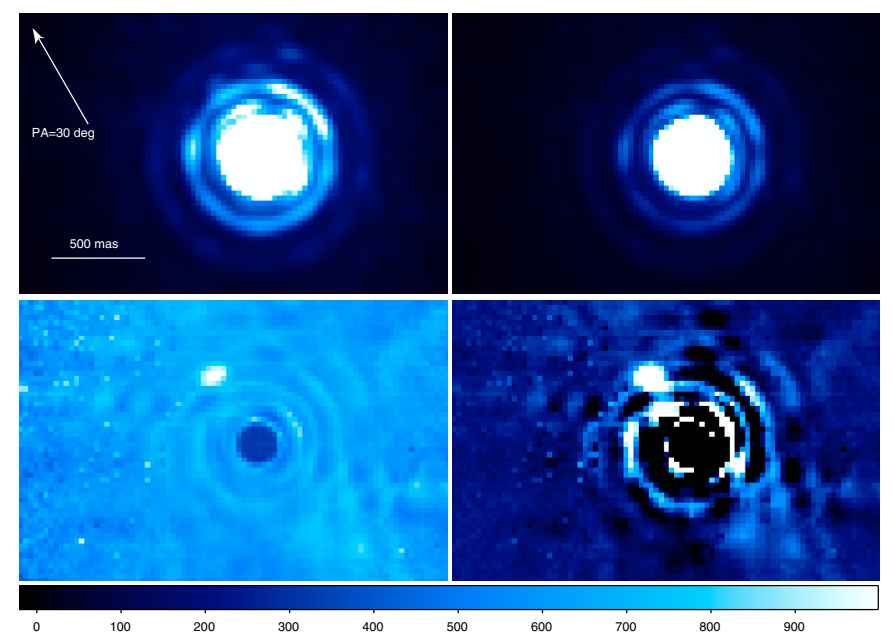

Fig. 1. $\beta$ Pic and HR 2435 recentered and saturated $L^{\prime}$ images (top left and top right, respectively) in data set A. Below are the divided (bottom left) and subtracted (bottom right) images. North is up and east to the left. A candidate companion is clearly detected at a PA of $\simeq 32^{\circ}$, i.e., along the NE side of the disk, at a separation of about $0{ }^{\prime} 41$ from the star.

- The last approach was actually to use the MISTRAL deconvolution algorithm (Mugnier et al. 2004), based on a maximum a posteriori scheme. Nevertheless, MISTRAL relies on a strict convolution process between image and reference, which is not the case for our saturated data. A first step is therefore to perform a posteriori correction of saturated parts of the image and reference. This is done using a simulated Airy pattern. The top of the Airy pattern replaces the image saturated pixels. The flux level is adjusted using the first Airy rings. Such a correction is possible because of the very good Strehl ratio on the image. Meanwhile, if this a posteriori correction does not significantly affect the restitution of the object structures, it could obviously degrade the relative photometry. The deconvolution process is thus an alternative approach (compared to reference subtraction and division) to image processing that is less sensitive to image-versusreference centering. It allows the best measurements of relative position with a precision of 0.3 pixels, but it remains uncertain for the relative photometry due to using saturated images.

\section{A candidate companion in the $\beta$ Pic disk?}

\subsection{Results from the highest quality data (set $A$ )}

Using three independent approaches, the companion detection is confirmed at the same location. The resulting images, using the maximum likelihood algorithm for recentering, are reported in Fig. 1. The companion candidate (hereafter, the $\mathrm{CC}$ ) point-like signal is clearly visible in the divided and subtracted images. The maximum of the signal is about 190 ADU. Different techniques (variable aperture photometry, 2D-Gaussian fitting, PSF-fitting) were used to extract the CC flux, giving consistent results. As the reference recentering and rescaling actually dominate the flux measurement precision, flux uncertainties were derived by considering respective variations of 0.3 pixel and $5 \%$ in the subtraction process. We obtain a contrast of $\Delta L^{\prime}=7.7 \pm 0.3$ between the $\mathrm{CC}$ and $\beta$ Pic. Using deconvolution, we derive a separation of $411 \pm 8$ mas and a PA of $31.8 \pm 1.3^{\circ}$ relative to the primary, i.e. along the NE side of the disk.

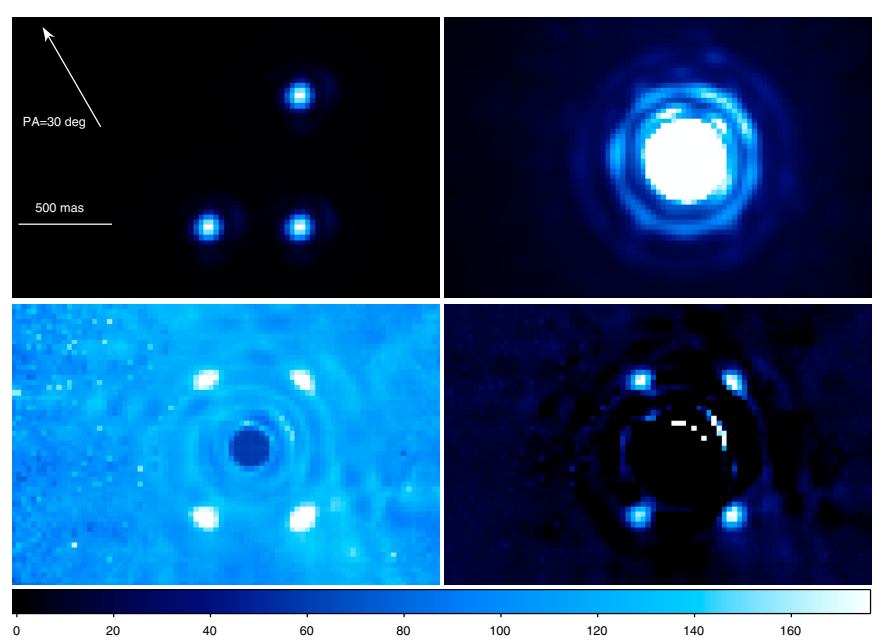

Fig. 2. Top left: simulated planets at PA of $150^{\circ}, 210^{\circ}$, and $330^{\circ}$. Top right: composite image of $\beta$ Pic plus the fake planets. Bottom left: division of the composite image by the saturated image of HR 2435. Bottom right: scaled subtraction of the composite image by the saturated image of HR 2435. Even a slight ( 0.3 pixel) relative offset between $\beta$ Pic and HR 2435 affects the resulting shape of the fake planets as much as the candidate one. In particular, triangular shapes can be observed, due to the proximity of the slightly inner Airy ring.

The use of different methods excludes artefacts created during the reduction process. In particular, the result of the deconvolution rules out any effect that could be introduced by imperfect estimation of the offset between $\beta$ Pic and HR 2435 saturated images due, for instance, to a possible contribution of the disk. We did check anyway that the disk signal is very faint and not significantly asymmetric. To rule out detector effects, we looked for possible remanence and electronic ghosts that could occur because our images are saturated. Inspection of individual images excludes any contamination by these two effects that rapidly disappear after a few frames. Artefacts due to the very good, but still imperfect, $\mathrm{AO}$ correction are still possible. However, aberrations due to a modulation of the deformable mirror would generally lead to either symmetrical or anti-symmetrical patterns. Static aberrations should be present just as around $\beta$ Pic and HR 2435, as both stars were observed with similar pupil configurations. We then tested the possible impact of an imperfect removal of static speckles due to the variation in the parallactic angle during the observations of $\beta$ Pic and HR 2435 (up to $8^{\circ}$ ). We processed individual pairs of data for $\beta$ Pic and HR 2435 taken with parallactic angles equal within $\pm 0.4^{\circ}$ and added up the individual subtracted images. The $\mathrm{CC}$ is still present and appears slightly sharper (but still compatible with the instrumental resolution). In addition, since the same signal is also observed a few nights apart (see below), we conclude that quasi-static aberrations are unlikely.

To further assess the reality of the detection and test the CC photometry, we added three "fake planets" at similar separations but different $\mathrm{PA}\left(150^{\circ}, 210^{\circ}\right.$, and $\left.350^{\circ}\right)$ to the recentered and stacked image of $\beta$ Pic. The fake planet images were generated by scaling and shifting an unsaturated image of $\beta$ Pic taken the same night. To match the level of the observed signal, the magnitudes of the fake planets were scaled to the measured flux ratio on set A. We then subtracted the scaled image of HR 2435 to that of the composite image. The result is shown in Fig. 2, from which it is clear that the fake planets produce similar features to the observed signal, supporting our contrast estimate. 


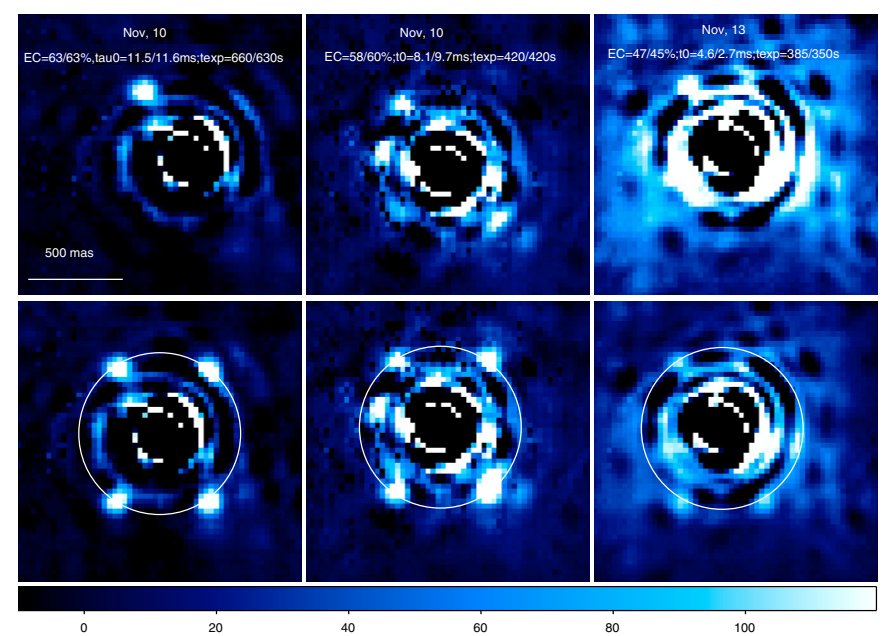

Fig. 3. Top: scaled subtraction of the composite image by the saturated image of HR 2435 for sets A-C (from left to right). Bottom: simulated planets at PA of $150^{\circ}, 210^{\circ}$, and $330^{\circ}$ for sets $\mathrm{A}-\mathrm{C}$.

We therefore derive an apparente magnitude of $L^{\prime}=11.2 \pm 0.3$ for the $\mathrm{CC}$.

\subsection{Results from lower quality data (sets $B$ and $C$ )}

All other sets of data were processed as before. It quickly appeared that the detection becomes more marginal when the exposure time and/or the image quality decrease. For the best remaining sets in terms of atmospheric conditions and relative parallactic angle between $\beta$ Pic and HR 2435 (described in Sect. 2.1 and Table 1), the results are shown in Fig. 3. The same point-like signal is seen in both cases, although with a lower signal-to-noise ratio. This is due to the lower exposure times and the (slightly) poorer image qualities. This is illustrated in Fig. 2, where we also added fake planets with magnitudes equal to that of the fake planets used for Set A to the data sets B and C. The fake planet signals are, like the $\mathrm{CC}$ signal, much less detectable under these conditions; hence the results from our 3 data sets are consistent.

\section{Bound companion or background object?}

With these data alone, it is not excluded that the CC could be a foreground or background object. To definitely test this hypothesis, second-epoch measurements are needed. Using the known $\beta$ Pic proper and parallactic motions, we considered the position of the $\mathrm{CC}$ assuming it would be a stationary contaminant. It would be closer than 0.2 to $\beta$ Pic in 2008 , hence not detectable with current techniques. In past years, $\beta$ Pic has been monitored by numerous programs of the Hubble Space Telescope, including observations using the Advanced Camera for Surveys (Golimowski et al. 2006; Kalas et al. 2005), the Near-Infrared Camera and Multi-Object Spectrometer (Brown et al. 1999), and the Wide Field Planetary Cameras (Kalas et al. 2000). The lack of spatial resolution or the size of the coronographic mask prevented getting any reliable hints of any point sources close to the star.

To our knowledge, the highest angular resolution and dynamical data available are the coronagraphic data taken by Heap et al. (2000) in 1997 September with the Space Telescope Imaging Spectrogrpah (STIS). These authors were able to probe the close stellar environment down to 0.75 from $\beta$ Pic. They did not report any $\mathrm{CC}$ in their images at the expected location of 0.9 North and 0.'25 East of $\beta$ Pic. Based on STIS detection limits and the disk brightness derived by Heap et al. (2000), all objects brighter than $V \leq 17$ would have been detected. Red giants or supergiants can be ruled out for their distances, which would bring them unrealistically far (typically $\gtrsim 10000 \mathrm{pc}$ ) from us. If we consider a foreground or background stellar field contaminant, it would therefore have a large color difference $(V-L) \geq 5.8$, i.e., a spectral type later than mid-M. Based on the number density of L dwarfs of $1.9 \times 10^{-3} \mathrm{pc}^{-3}$ given by Burgasser (2001) and Cruz et al. (2003), the probability of finding a foreground or background field L dwarf in a region of 500 mas radius around $\beta$ Pic and with $L^{\prime}=11.2$ is about $10^{-10}$. Without any assumption on the contaminant spectral type, one can use galactic population model outputs to estimate the probability of finding any $\left(L^{\prime} \leq 12\right)$ galactic source at 500 mas from $\beta$ Pic. We find a low probability of $6 \times 10^{-5}$. Last, we cannot strictly rule out a contamination by an extragalactic source, such as a high-redshift quasar. In conclusion, a contamination appears very unlikely. In addition, that the candidate companion falls into the disk strongly favors it being bound to the star.

\section{Implications for understanding the $\beta$ Pic system}

With an $L^{\prime}$ magnitude of $11.2 \pm 0.3$, and assuming a distance of $19.3 \pm 0.2 \mathrm{pc}$ and an age of $12_{-4}^{+8}$ Myr (Zuckerman et al. 2001), the mass of the CC is estimated to $9_{-2}^{+3} M_{\text {Jup }}$ using COND models (Baraffe et al. 2003) and $8_{-2}^{+4} M_{\text {Jup }}$ using DUSTY models (Chabrier et al. 2000). The planet should still be in the phase of cooling: Dusty and COND models predict effective temperatures of 1400 and $1600 \mathrm{~K}$, respectively. The validity of these models in the case of young planets formed by core accretion has recently been questioned (Marley et al. 2007) on the basis that the accretion shock might affect the planet initial internal entropy and its subsequent early thermal evolution. These authors claim that young giant planets could be significantly cooler and fainter than predicted so far. However, the treatment of the accretion shock is still a matter of debate. The impact of the initial disk mass also needs to be studied. This may be important in the present case, as $\beta$ Pic is significantly more massive than the Sun. In the present case, the Marley et al. (2007) model predicts a luminosity ten times fainter than what we observed for an $8 M_{\text {Jup }}$ planet at the age of $\beta$ Pic. However, we can already note that a companion at a true separation of $\sim 8$ AU could not be significantly more massive than 10-20 $M_{\text {Jup }}$, since otherwise it would have been detected through radial velocity measurements, as shown by a detailed analysis to be presented in a forthcoming paper (Desort et al., in preparation).

If the observed projected separation happens to be the physical one or one close to the physical one, then the companion explains three very intriguing and so far unique characteristics of the $\beta$ Pic system: the warp in the inner disk, the inner belts, and the falling evaporating bodies (Freistetter et al. 2007). Mouillet et al. (1997) and Heap et al. (2000) show that the warp of the disk constrains the (mass $M_{\mathrm{p}}$, semi-major axis $a$ ) domain of the planet as

$\log \left(M_{\mathrm{p}} / M_{\star}\right)+2 \log a+\log t \approx 6.7$,

where $M_{\star}=1.8 M_{\odot}$ (Crifo et al. 1997) and $t$ is the age of the star. Given an age $t=12 \mathrm{Myr}$, masses $M_{\mathrm{p}}=6$ and $13 M_{\text {Jup }}$ give $a=$ 9.7 and 7.6 AU, respectively, nicely bracketting the measured projected separation. This further strengthens the likelihood that the observed CC is the planet causing the warp. 
At the time of the submission of this letter, this companion was to our knowledge the first one possibly detected around an A-type main sequence star. Recent results published since then by Marois et al. (2008) and Kalas et al. (2008) also show the presence of planets at distances 24 to 118 AUs from two A-type stars. The companion we imaged around $\beta$ Pic could nevertheless be the first extrasolar planet ever imaged so close to its parent star: 8 AU. In particular, it would be located well inside the orbits of the outer planets of the Solar System. Its proximity and location inside the $\beta$ Pic disk suggest a formation process by core accretion or disk instabilities rather than the binary-like formation mechanisms proposed for the companions to $2 \mathrm{M} 1207$ and AB Pic (Chauvin et al. 2005a,b). More direct imaging observations should allow the planet orbit to be constrained, hence the planet's dynamical mass to be measured. This would in turn allow testing of the models of planet formation. This is dramatically needed as the evolutionary models are not calibrated with real data in these ranges of masses and ages. Finally, we note that, unfortunately, if the projected separation is the physical one, the planet orbital period is about 16 years and should be much closer to the star at the present time so undetectable with $\mathrm{NaCo}$.

Acknowledgements. We acknowledge financial support from the French Programme National de Planétologie (PNP, INSU), as well as from the French Agence Nationale pour la Recherche (ANR; project NT05 - 444463). These results have made use of the SIMBAD database, operated at the CDS, Strasbourg, France. We also thank the ESO staff for its help during the NaCo observations. We thank J.-C. Augereau for useful discussions of the $\beta$ Pic disk. AML thanks also P. Rubini for his help on the layout of the paper. Finally, we thank the referee for his rapid and precious comments on the paper.

\section{References}

Augereau, J.-C., Nelson, R. P., Lagrange, A.-M., Papaloizou, J. C. B., \& Mouillet, D. 2001, A\&A, 370, 447

Baraffe, I., Chabrier, G., Barman, T. S., Allard, F., \& Hauschildt, P. H. 2003, A\&A, 402, 701

Beust, H., \& Morbidelli, A. 2000, Icarus, 143, 170

Brown, J., Bennum, D., Schultz, A. B., et al. 1999, BAAS, 31, 1487

Burgasser, A. 2001, Ph.D. Thesis, California Institute of Technology

Chabrier, G., Baraffe, I., Allard, F., \& Hauschildt, P. H. 2000, ApJ, 542, 464
Chauvin, G., Lagrange, A.-M., Dumas, C., et al. 2005a, A\&A, 438, L25 Chauvin, G., Lagrange, A.-M., Zuckerman, B., et al. 2005b, A\&A, 438, L29 Crifo, F., Vidal-Madjar, A., Lallement, R., Ferlet, R., \& Gerbaldi, M. 1997, A\&A, 320, L29

Cruz, K. L., Reid, I. N., Liebert, J., Kirkpatrick, J. D., \& Lowrance, P. J. 2003, AJ, 126, 2421

Freistetter, F., Krivov, A. V., \& Löhne, T. 2007, A\&A, 466, 389

Devillard, N. 1997, Messenger, 87

Ducati, J. R., Bevilacqua, C. M., Rembold, S. B., \& Ribeiro, D. 2001, ApJ, 558, 309

Galland, F., Lagrange, A.-M., Udry, S., et al. 2006, A\&A, 447, 355

Golimowski, D. A., Ardila, D. R., Krist, J. E., et al. 2006, AJ, 131, 3109

Gorkavyi, N., Heap, S., Ozernoy, L., Taidakova, T., \& Mather, J. 2004, in Planetary Systems in the Universe, ed. A. Penny, Proc. IAU Symp., 202, 331 Gratadour, D., Mugnier, L. M., \& Rouan, D. 2005, A\&A, 443, 357

Heap, S. R., Lindler, D. J., Lanz, T. M., et al. 2000, ApJ, 539, 435

Lecavelier des Etangs, A., Deleuil, M., Vidal-Madjar, A., et al. 1995, A\&A, 299, 557

Kalas, P., \& Jewitt, D. 1995, AJ, 110, 794

Kalas, P., Larwood, J., Smith, B. A., \& Schultz, A. 2000, ApJ, 530, L133

Kalas, P., Graham, J. R., \& Clampin, M. 2005, Nature, 435, 1067

Kalas, P., Graham, J. R., Chiang, E., et al. 2008, Science [arXiv: 0811. 1994]

Krist, J. E., Burrows, C. J., Stapelfeldt, K. R., Watson, A. M., \& WFPC2 Investigation Definition Team 1995, A\&AS, 187, 4413

Lafrenière, D., Doyon, R., Marois, C., et al. 2008, ApJ, 670, 1367

Lagrange, A.-M., Backman, D. E., \& Artymowicz, P. 2000, Planetary Material around Main-Sequence Stars, in Protostars and Planets IV, ed. V. Mannings, A. P. Boss, \& S. S. Russell (Tucson: University of Arizona Press), 639

Leggett, S. K., Golimowski, D. A., Fan, X., et al. 2002, ApJ, 564, 452 Lenzen, R., Hartung, M., Brandner, W., et al. 2003, SPIE, 4841, 944

Luhman, K. L., Wilson, J. C., Brandner, W., et al. 2006, ApJ, 649, 894

Metchev, S., \& Hillenbrand, L. 2006, ApJ, 651, 1166

Mugnier, L. M., Fusco, T., \& Conan, J.-M. 2004, JOSAA, 21, 1841

Marley, M. S., Fortney, J. J., Hubickyj, O., Bodenheimer, P., \& Lissauer, J. J. 2007, ApJ, 655, 541

Marois, C., MacIntosh, B., Barman, T., et al. 2008, Science [arXiv: 0811.2606]

Mayor, M., \& Queloz, D. 1995, Nature, 378, 355

Mouillet, D., Larwood, J. D., Papaloizou, J. C. B., \& Lagrange, A.-M. 1997, MNRAS, 292, 896

Okamoto, Y. K., Kataza, H., Honda, M., et al. 2004, Nature, 431, 660

Rousset, G., Lacombe, F., Puget, P., et al. 2003, SPIE, 4839, 140

Smith, B., \& Terrile, R. 1984, Science, 226, 1421

Telesco, C. M., Fisher, R. S., Wyatt, M. C., et al. 2005, Nature, 433, 133

Thébault, P., \& Beust, H. 2001, A\&A, 376, 621

van Dessel, E., \& Sinachopoulos, D. 1993, A\&AS, 100, 517

Woodgate, B. E., Kimble, R. A., Bowers, C. W., et al. 1998, PASP, 110, 1183

Zuckerman, B., Song, I., Bessel, M. S., \& Webb, R. A. 2001, ApJ, 562, L87 